\title{
A SWIMMING POOL-ASSOCIATED OUTBREAK OF CRYPTOSPORIDIOSIS IN STAFFORDSHIRE, ENGLAND, OCTOBER TO DECEMBER 2007
}

\author{
N Coetzee (nic.coetzee@hpa.org.uk) ${ }^{1}, 0$ Edeghere ${ }^{1}$, J M Orendi², R Chalmers ${ }^{3}$, L Morgan ${ }^{1}$ \\ 1. Health Protection Agency, West Midlands North, United Kingdom \\ 2. University Hospital of North Staffordshire, National Health Service (NHS) Trust, Stoke-on-Trent, United Kingdom \\ 3. UK Cryptosporidium Reference Unit, National Public Health Service (NPHS) Microbiology, Swansea, United Kingdom
}

\begin{abstract}
In October 2007 an increase in laboratory-confirmed cryptosporidiosis cases in Staffordshire, England prompted an outbreak investigation. Case ascertainment included interviewing suspected cases and contacts and obtaining faecal specimens from those with diarrhoea for laboratory identification. Over a three-month period we identified 57 cases of cryptosporidiosis (39 confirmed) distributed across 36 households. The majority of cases (69\%) were younger than 20 years. The most plausible exposure was multiple swimming episodes (56\% of cases) in 13 local public swimming pools. One large swimming pool was most frequently visited by swimmers and considered a significant contributor to transmission because of substandard filtration and maintenance systems. Control measures focused on inspecting and improving operating standards at swimming pools, hygiene information to swimmers, and early detection and exclusion of cases. The rapid case investigation described in this paper provided adequate information for the early detection and control of a typical seasonal swimming pool related cryptosporidiosis outbreak. Ensuring adequate filtration standards at public swimming pools particularly before the high use periods of late summer and autumn remains a priority.
\end{abstract}

\section{Introduction}

The improvement of water treatment systems in England and Wales has resulted in fewer drinking water-related cryptosporidiosis outbreaks in recent years [1]. By contrast, swimming poolassociated outbreaks continue to occur, with incidence peaking in late summer and autumn when swimming pool use is highest [2]. Outbreaks linked to interactive water features have also increased in prominence [3].

In November 2007 laboratory surveillance indicated a fourfold increase of cryptosporidiosis cases in northern Staffordshire, England, compared to 2006 data (16 vs. 4 cases). Routine questioning of cases by environmental health officers revealed all had recent public swimming pool exposures. We undertook a rapid case investigation aimed at targeting timely and appropriate control measures.

Methods

The University Hospital North Staffordshire microbiology laboratory serves the northern Staffordshire population consisting of approximately 500,000 residents.
A confirmed case of cryptosporidiosis was defined as any northern Staffordshire resident with diarrhoea confirmed by the detection of Cryptosporidium oocysts in a stool sample by microscopic examination, from 15 October to 24 December 2007.

A probable case was defined as any household or close contact of a confirmed case presenting with watery diarrhoea or diarrhoea plus abdominal cramps with nausea and/or vomiting from 15 October to 24 December 2007.

An outbreak management team consisting of public health investigators, microbiologists, environmental health officers, and a media officer was convened to oversee the investigation and the implementation of control measures. We alerted local general practitioners and acute care hospital practitioners to be vigilant and encourage confirmatory testing of suspected cases, and to give patients appropriate hygiene and exclusion advice. Public health officers used a standardised questionnaire to interview the cases in person or over the telephone. Children were interviewed with an adult family member present. Exposure data included sources of drinking water, recreational water exposure including swimming, food consumption, animal contact and recent travel. Cases and their close contacts were given detailed advice on hygiene measures, exclusion from work or school if indicated, and exclusion from swimming until 14 days after last symptoms [4].

Further probable cases were identified through the investigation of family members and close contacts of cases, and encouraging those with symptoms to submit faecal samples.

Laboratory and interview data were captured anonymously in a line listing and analysed descriptively using EPIData statistical software (Version 2) [5].

Swimming pools identified during questioning of cases were inspected by environmental health officers against the standards laid down by the Pool Water Treatment Advisory Group (PWTAG) [6]. Water samples were not taken from individual pools for Cryptosporidium testing, as control interventions were implemented without delay based on pool inspection results.

Primary laboratory diagnosis was based on the demonstration of Cryptosporidium oocysts in stool specimen, using the modified Ziehl-Neelsen stain [7]. A number of samples positive for oocysts 
were submitted for confirmation and species identification at the UK Cryptosporidium Reference Unit. Oocysts separated from faecal debris by saturated salt flotation were disrupted at $100{ }^{\circ} \mathrm{C}$ for 60 minutes, digested with proteinase $\mathrm{K}$ in lysis buffer at $56{ }^{\circ} \mathrm{C}$ and deoxyribonucleic acid (DNA) extracted by spin-column filtration (QiaAMP DNA mini kit, Qiagen) [8]. DNA was routinely subjected to polymerase chain reaction-restriction fragment length polymorphism (PCR-RFLP) of the Cryptosporidium oocyst wall protein (COWP) gene in the first instance and a subset confirmed by PCR-RFLP using nested primer sets for the small subunit ribosomal ribonucleic acid (SSU rRNA) gene $[9,10]$.

\section{Results}

Thirty nine confirmed cases were identified. Their median age was 13 years with a male: female ratio of 1.2 . Twenty seven (69\%) cases were younger than 20 years with males (18/27) predominating (Figure 1). The distribution of symptom onset dates for confirmed cases is given in Figure 2. Confirmed cases were distributed across 36 households. An additional 18 probable cases were identified in these 36 households yielding a total of 57 cases. Three households each had a second confirmed case representing likely transmission between siblings.

Six confirmed cases (three of them less than 15 years old) were admitted to hospital for treatment.

With the help of the two water companies supplying domestic water to northern Staffordshire we were able to confirm that cases were distributed over several separate raw water supply and quality zones. Domestic water could therefore be excluded as a potential source of infection at the outset of the investigation. Confirmed cases reported no swimming in open water sources (rivers, ponds). Cases had minimal exposure to other non-swimming pool potential sources of infection: three had visited a zoo or livestock farm; three had contact with a sick pet; and seven reported recent travel abroad.

Twenty two (56\%) confirmed cases reported recent swimming at one or more of the 13 local swimming pools prior to onset of symptoms. The median time from exposure to swimming pool water to onset of symptoms was seven days (range 1 to 25 days), representing an approximate incubation period. These 22 swimming pool-exposed cases were distributed across 20 households, with two households each having a second case. Eight cases confirmed swimming in more than one swimming pool, others frequented one place only. Pool A (a large and very busy water theme park) was visited on at least one occasion by 14 cases. Eight of these 14 cases swam exclusively at Pool A. Thirteen of the cases with pool A exposure attended weekend swimming disco or Halloween parties organised at the pool between 26 October and 17 November 2007.

Faecal samples from 22 cases (including nine with swimming pool exposure) were submitted for species identification. Four were Cryptosporidium parvum (of which one case had swimming pool exposure but not at pool A); and 18 were Cryptosporidium hominis (of which eight had swimming pool exposure - four exclusively at pool $A$ and four at other pools). Three of the cases with no swimming exposure in which $\mathrm{C}$. hominis was identified were preschool siblings of cases with the same infection and swimming exposure at pool $A$.

In 17 cases (13 reporting swimming pool exposure) species identification was not requested and a meaningful analysis of species type association with individual swimming pools could not be undertaken.

Pool A was inspected during the last week of November 2007 and found not to have adequate sand filtration depth, continuous coagulant dosing, flow rate monitoring, and backwashing routines. Policies for pool evacuation and decontamination following faecal incidents were not in place. Urgent remedial measures were instigated and hygiene conditions improved. Subsequently, all other public and school swimming pools were also inspected and found to have adequate filtration standards but variable faecal incident policy standards and uptake. Bacteriological standards at all 13 swimming pools were within acceptable limits.

\section{Discussion and conclusion}

This outbreak of 39 confirmed and 18 probable cases of cryptosporidiosis had a significant impact on the affected population and households in terms of hospitalisation and absence from schooling or work. We believe that swimming parties at pool A, coupled with ineffective filtration systems and large numbers of

\section{F I G U R E 1}

Age and sex distribution of confirmed cryptosporidiosis cases, Staffordshire, England, October to December $2007(\mathbf{n}=39)$

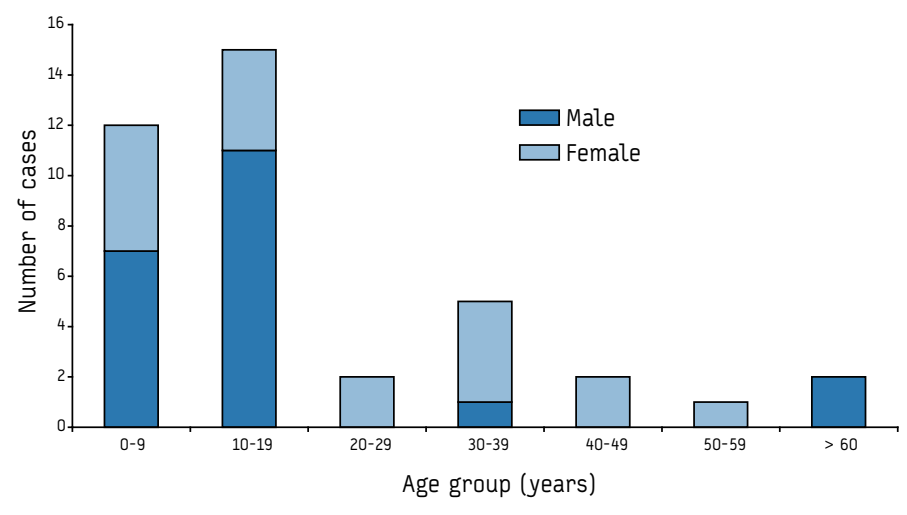

F I G U R E 2

Confirmed cryptosporidiosis cases, by swimming pool exposure and date of onset of symptoms, Staffordshire, England, October to December $2007(n=39)$

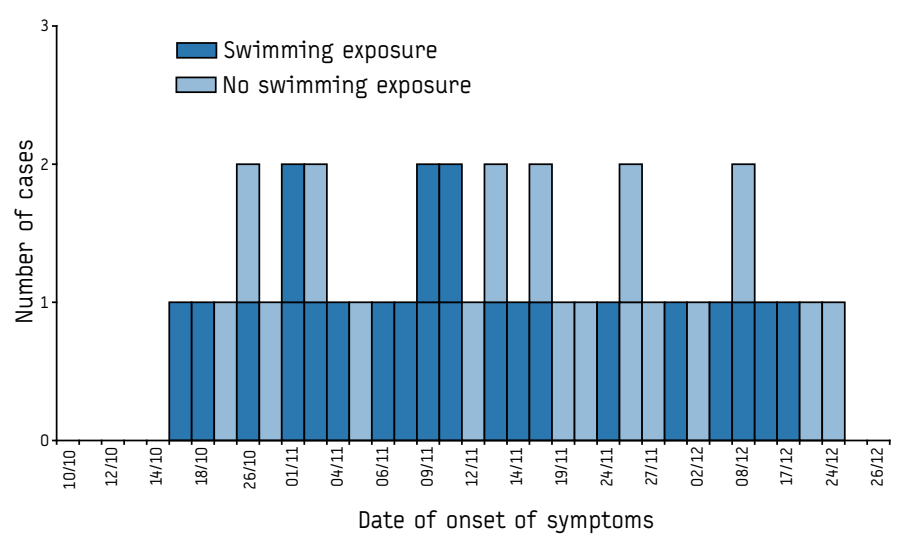


visitors, contributed to the early part (first 5 weeks) of the outbreak. Not surprisingly, the incidence of infection was highest in younger age groups who swam often and at a variety of different swimming pools. Although difficult to verify due to sampling limitations, C. hominis was likely to be most associated with swimming pool exposure during the initial stages of the outbreak. Secondary household transmission contributed to the size of the outbreak and was probably underreported. The role of travel exposure appeared to be limited but had been an important factor at the onset of other similar outbreaks [11].

Outbreaks associated with several swimming pools are often prolonged and difficult to investigate due to multiple exposures and incomplete case ascertainment $[11,12]$. We were limited in our ability to fully investigate the contribution of other exposures, such as private swimming pools and common food sources, that could have accounted for some cases. It is likely that more severe cases were overrepresented in this outbreak. Despite this limitation, the laboratory based surveillance system proved reliable in detecting the outbreak. Coupled with rapid case investigation, we were able to identify public swimming pool exposure as the most likely cause of the outbreak and implement control measures. Improved hygiene measures at Pool A could not be implemented early enough in the outbreak to impact on disease incidence, but are in place for the next season.

Developing a pre-emptive approach to seasonal swimming pool-associated Cryptosporidium outbreaks is clearly feasible and important. The means for detection, prevention and control are readily available although often not implemented in time [11]. The existing guidance published by PWTAG should be followed and audited by swimming pool operators and local authorities to ensure adequate filtration systems, maintenance standards, and hygiene policies are in place well before the summer months $[6,13]$. One example of an auditing framework is that provided by the Institute of Sport and Recreation Management National Pool Safety Award [14]. Public health units are in a strong position to closely monitor Cryptosporidium incidence in anticipation of the seasonal swimming-related peak, and to rapidly communicate advice to clinicians and appropriate health messages to schools and the public.

\section{Acknowledgements}

We are grateful for the support we received from the local environmental health departments, the Health Protection Agency laboratories, and the UK Cryptosporidium Reference Unit for technical and scientific support.

\section{References}

1. Smith A, Reacher M, Smerdon W, Adak GK, Nichols G, Chalmers RM. Outbreaks of waterborne infectious intestinal disease in England and Wales, 1992-2003. Epidemiol Infect. 2006;134(6):1141-9.

2. Nichols G, Chalmers R, Lake I, Sopwith W, Regan M, Hunter P, Grenfell P, Harrison F, Lane C. Cryptosporidiosis: A report on the surveillance and epidemiology of Cryptosporidium infection in England and Wales. Drinking Water Directorate; 2006 Sept. Contract No.: DWI 70/2/201. Sponsored by the Water Directorate of the Department of Environment Food and Rural Affairs. Available from: http://www.dwi.gov.uk/research/reports/DWI70_2_201.pdf

3. Jones M. Boccia D, Kealy M, Salkin B, Ferrero A, Nichols G, Stuart JM. Cryptosporidium outbreak linked to interactive water feature, UK: importance of guidelines. Euro Surveill. 2006;11(4):pii-612. Available from: http://www. eurosurveillance.org/ViewArticle.aspx?Articleid $=612$
4. Working Group of the PHLS Advisory Group on Gastrointestinal Infections. Preventing person-to-person spread following gastrointestinal infections: guidelines for public health physicians and environmental health officers. Commun Dis Publ Health 2004;7(4):362-384. Available from: http://www.hpa. org.uk/cdph/issues/CDPHvol7/No4/guidelines2_4_04.pdf

5. Bennett S, Myatt M, Jolley D, Radalowicz A. Data management for surveys and trials. A practical primer using EpiData. Available from: http://www.epidata. dk/downloads/dmepidata.pdf

6. Pool Water Treatment Advisory Group. Swimming Pool Water: treatment and quality standards. Greenhouse Publishing, 1999.

7. Health Protection Agency. Investigation of specimens other than blood for parasites. National Standard Method BSOP 31, Issue 3, 2008. Available online: http://www.hpa-standardmethods.org.uk/documents/bsop/pdf/bsop31.pdf

8. Elwin K, Chalmers RM, Roberts R, Guy EC, Casemore DP. The modification of a rapid method for the identification of gene-specific polymorphisms in Cryptosporidium parvum, and application to clinical and epidemiological investigations. Appl Environ Microbiol. 2001;67(12):5581-4.

9. Spano F, Putignani L, McLauchlin J, Casemore DP, Crisanti A. PCR-RFLP analysis of the Cryptosporidium oocyst wall protein (COWP) gene discriminates between C. wrairi and C. parvum, and between C. parvum isolates of human and animal origin. FEMS Microbiol Lett. 1997;150(2):209-17.

10. Xiao L, Alderisio K, Limor J, Royer M, Lal AA. Identification of species and sources of Cryptosporidium oocysts in storm waters with a smallsubunit rRNA-based diagnostic and genotyping tool. Appl Environ Microbiol. 2000;66(12):5492-5498.

11. Nichols G. Cryptosporidiosis associated with swimming pools in England. Euro Surveill. 1999;3(48):pii=1249. Available from: http://www.eurosurveillance.org./ ViewArticle.aspx?Articleid $=1294$

12. Puech MC, McAnulty JM, Lesjak M, Shaw N, Heron L, Watson JM. A statewide outbreak of cryptosporidiosis in New South Wales associated with swimming at public pools. Epidemiol Infect. 2001;126(3):389-396.

13. Pool Water Treatment Advisory Group. Cryptosporidium in water supplies advice for pool operators, 2001. Available from: http://www.pwtag.org/home. html

14. Institute of Sport and Recreational Management. National Pool Safety Award. [homepage on Internet] Available from: http://www.isrm.co.uk/education/ pool_award_national.html

This article was published on 6 November 2008

Citation style for this article: Coetzee N, Edeghere 0, Orendi JM, Chalmers R, Morgan L. A swimming pool-associated outbreak of cryptosporidiosis in Staffordshire, England October to December 2007. Euro Surveill. 2008:13(45):pii=19028. Available online: $\mathrm{http}: / /$ www.eurosurveillance.org/ViewArticle.aspx?ArticleId $=19028$ 